\title{
Functionalization of 3-D porous thermoplastic polyurethane scaffolds by two-stage polydopamine/hydroxyapatite composite nanoparticles
}

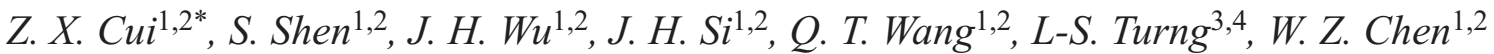 \\ ${ }^{1}$ School of Materials Science and Engineering, Fujian University of Technology, 350118 Fujian, China \\ ${ }^{2}$ Fujian Provincial Engineering Research Center of Die \& Mold, Fujian University of Technology, 350118 Fujian, China \\ ${ }^{3}$ Wisconsin Institutes for Discovery, University of Wisconsin-Madison, Madison, 53715 Wisconsin, United States \\ ${ }^{4}$ Department of Mechanical Engineering, University of Wisconsin-Madison, Madison, 53706 Wisconsin, United States
}

Received 11 November 2019; accepted in revised form 21 January 2020

\begin{abstract}
In this work, the polydopamine (PDA) coating layer was first applied to modify hydroxyapatite (HA) nanoparticles and then the functional PDA/HA (pHA) composite nanoparticles were obtained. Subsequently, a 3-D porous thermoplastic polyurethane (TPU) scaffold modified by two-stage pHA nanoparticles ( $\mathrm{pHA}-\mathrm{TPU} / \mathrm{pHA}$ ) was fabricated by vacuum-assisted solvent casting and ultrasonication-assisted methods. The results demonstrated that the pHA nanoparticles were successfully prepared and introduced into the pHA-TPU/pHA composite scaffold both internally and externally. The pHA-TPU/pHA composite scaffold had an excellent interconnected network structure with high porosity of $85.7 \%$ and large pore sizes ranging from 100-500 $\mu \mathrm{m}$. The hydrophilicity and comprehensive mechanical properties of the pHA-TPU/pHA composite scaffold were significantly higher than those of the TPU scaffold due to the addition of the pHA nanoparticles. The attachment and viability of mouse embryonic osteoblast cells (MC3T3-E1) cultured on the pHA-TPU/pHA composite scaffold were also higher than those on the TPU scaffold. These results suggest that the functional pHA-TPU/pHA composite scaffold has superior mechanical and biological properties, which makes it potentially useful for tissue engineering scaffolds.
\end{abstract}

Keywords: polymer composites, polydopamine coating, 3-D porous scaffold, thermoplastic polyurethane, hydroxyapatite

\section{Introduction}

Tissue engineering is an evolving interdisciplinary field that aims to repair, renovate, or promote tissue function in concert with progenitor cells and biolog$\mathrm{ical} /$ chemical stimuli $[1,2]$. Bone tissue engineering has received increasing attention because of the presence of a wide range of bone diseases, such as trauma, tumors, or other damage. From the viewpoint of the material component, bone tissue is a natural composite that consists of three elements: (1) nano-hydroxyapatite (HA), (2) nanofibrous collagen matrix, and (3) organic matter that can be dissolved in water $[3,4]$. Consequently, various types of multi-phasic or multi-layered scaffolds have been prepared and used as temporary bone substitutes via mimicking the natural structure of bone tissue. The ideal threedimensional (3-D) porous scaffold can be applied to mimic or replicate the natural bone extracellular matrix $(\mathrm{ECM})$ and to promote the reconstruction of bone tissues [5].

Thermoplastic polyurethane (TPU), as a class of versatile elastomers, consists of hard and soft segments composed of amorphous and pseudo-crystalline structures, respectively. For that reason, TPU possesses many advantages, such as controllable mechanical properties, tunable chemical structures, stable 
chemical properties, and good fatigue resistance and corrosion resistance [6]. As such, it has been extensively applied in tissue engineering fields to repair and regenerate tissues. Although TPU has excellent comprehensive performance, it also has the disadvantages of being bio-inert and super-hydrophobic, which restricts its application [7]. To overcome these shortcomings, bioactive inorganic nanoparticles have been widely applied as a filler in combination with TPU to accelerate osteoblast adhesion, proliferation, and osteointegration [8-11]. In particular, hydroxyapatite (HA) as a bioactive material has been frequently used in bone tissue engineering due to its high similarity in chemical and crystal structure to the mineral components of natural bone [12].

Various TPU/HA composite scaffolds have been reported as a promising application prospect in bone tissue engineering [13-15]. However, although the application of TPU/HA composite scaffolds in bone healing has been extensively explored, challenges remain concerning poor exposure of HA nanoparticles in the TPU matrix, resulting in poor contact between the bioactive filler and the cells, as well as the weak adhesive forces between the TPU matrix and the HA filler $[16,17]$ that might result in the implant loosening and, ultimately, implant failure [18]. Thus, it is necessary to develop a 3-D composite scaffold that can mimic the material component, structure, and mechanical properties of natural bone ECM.

To solve the poor interaction between HA nanoparticles and cells, in our previous study [19], we have prepared the porous 3-D HA-decorated TPU scaffold by using a novel and simple ultrasonic method. The cell biocompatibility of HA-decorated TPU scaffold was significantly improved because most of HA nanoparticles were exposed to the surface of the scaffold. However, there is still a problem in poor interfacial binding force between HA nanoparticles and TPU matrix. Due to the advantages of polydopamine (PDA), which not only can easily adhere onto virtually all types of substrates in an alkaline solution without complex linking agents [20,21], but also possesses excellent adhesiveness, hydrophilicity and biocompatibility [22], it has been used as a glue-like layer to solve the interface problem of HA-decorated TPU scaffold in our previous study [23]. The mechanical properties and cell biocompatibility of PDA/HA/ TPU scaffold were obviously increased due to the introduction of PDA coating.
Thus, one of the major purposes of this study was to generate a PDA coating layer on the surface of HA nanoparticles to obtain PDA/HA composite nanoparticles (pHA), and then to blend the pHA nanoparticles with the TPU to obtain a 3-D porous TPU/pHA composite scaffold. The interfacial bonding force between the TPU matrix and the pHA filler is expected to improve significantly due to the introduction of the PDA coating layer on the surface of HA particles. And another of the major purposes of this study was to design a 3-D porous TPU scaffold with two-stage pHA-nanoparticle modification; that is, pHA nanoparticles that coexist inside and outside of the TPU matrix. Thus, in the early stage, the outside pHA nanoparticles are easy to come into contact with and interact with cells to promote adhesion and proliferation. In the later stage, the inside pHA nanoparticles are continually released from the TPU matrix, which can continue to keep promoting the differentiation and formation of bone tissue. To develop a pHA-TPU/ pHA scaffold with a multi-layer structure is highly anticipated in the bone regeneration field. Up until now, such a scaffold has not been reported.

Herein, we report a novel 3-D porous pHA-TPU/ pHA scaffold with a two-stage modification layer. First, the pHA nanoparticles were successfully fabricated by coating PDA onto the surface of HA nanoparticles. Second, TPU/pHA composite scaffolds were prepared by a vacuum-assisted solvent casting method, which transferred the pHA nanoparticles inside the TPU matrix to increase their release time from the TPU matrix. Finally, the pHA-TPU/pHA composite scaffolds were fabricated via an ultrasonic technique that then transferred the pHA nanoparticles outside the TPU matrix to maximize exposure to cells. The morphology, thermal behavior, hydrophilicity, cell viability, and static and dynamic mechanical properties of TPU, TPU/pHA, and pHATPU/pHA composite scaffolds were systematically investigated.

\section{Materials and methods \\ 2.1. Materials}

Thermoplastic polyurethane (TPU, Tecoflex EG80A) was purchased from Noveon, Inc., USA. Hydroxyapatite nanoparticles (HA) with a diameter of about $20 \mathrm{~nm}$ were purchased from Nanjing Emper Nano Material Co, Ltd., China. Sodium chloride particles $(\mathrm{NaCl}$, food-grade) were purchased from 
Jiangsu Salt Industry Group Co., Ltd., China. $\mathrm{NaCl}$ particles with a size of 300 to $425 \mu \mathrm{m}$ were sifted out before use. Dopamine and tris(hydroxylmethyl) aminomethane (Tris) were obtained from Shanghai Aladdin Bio-Chem Technology. The anhydrous 1,4dioxane and ethanol were purchased from Guang Zhou Jinhuada Chemical Reagent Co. Ltd., China. The mouse embryonic osteoblast cells (MC3T3-E1) were purchased from Shanghai Gefan Biotechnology Co., Ltd., China.

\subsection{Preparation of $\mathbf{p H A}$ nanoparticles}

First, a $10 \mathrm{mM}$ Tris- $\mathrm{HCl}$ buffer solution was prepared and its $\mathrm{pH}$ was adjusted to 8.5. Then, $2 \mathrm{~g}$ of dopamine particles were added to the above-prepared $100 \mathrm{ml}$ solution to obtain a $2 \mathrm{~g} / 1$ dopamine solution. Next, $1 \mathrm{~g}$ of HA particles was immersed into the above-mentioned dopamine solution, followed by continuous stirring and sonication under ambient temperature for $24 \mathrm{~h}$. Finally, the pHA nanoparticles were collected by centrifugation at a speed of $8000 \mathrm{rpm}$, washed by deionized water to remove the unattached PDA, and then freeze-dried for further application.

\subsection{Preparation of 3-D porous TPU/pHA and pHA-TPU/pHA composite scaffolds}

First, $0.48 \mathrm{~g}$ of pHA nanoparticles were added to $60 \mathrm{ml}$ of a 1,4-dioxane/water (17/3) solvent mixture and sonicated for $30 \mathrm{~min}$ to obtain a homogeneous pHA suspension solution. Then $4.8 \mathrm{~g}$ of TPU particles were dissolved into the above-prepared solution under continuous stirring at $60^{\circ} \mathrm{C}$ for $12 \mathrm{~h}$ to obtain a uniform $8 \mathrm{wt} \%(\mathrm{w} / \mathrm{v})$ TPU/pHA blend solution (weight ratio of TPU/pHA was 100/10, w/w). The TPU/pHA blend solution was cast into an assembled $\mathrm{NaCl}$ template with the help of vacuum-assisted equipment. The detailed vacuum-assisted solvent casting procedure for fabrication of porous 3-D scaffolds was reported in our previous study [24]. The fabricated TPU/pHA/NaCl construct was pre-frozen at $-80^{\circ} \mathrm{C}$ for $12 \mathrm{~h}$ by deep refrigeration and then phase-separated by immersing it into an ethanol bath at $-20^{\circ} \mathrm{C}$ for $3 \mathrm{~d}$ to exchange the 1,4 -dioxane solvent. After solvent extraction, the formed TPU/pHA/ $\mathrm{NaCl}$ construct was dried by a vacuum oven to remove the ethanol and then leached in deionized water for $48 \mathrm{~h}$. The leached 3-D porous TPU/pHA composite scaffold was dried by freeze-drying for further application. For reference, the 3-D porous
TPU scaffold was also fabricated using the abovementioned process.

First, $0.3 \mathrm{~g}$ of pHA nanoparticles were put into $60 \mathrm{ml}$ deionized water and sonicated for $30 \mathrm{~min}$ to obtain $0.5 \mathrm{wt} \%(\mathrm{w} / \mathrm{v}) \mathrm{pHA}$ suspension solution. Then, the prepared 3-D porous TPU/pHA composite scaffolds were immersed into the above-prepared pHA homogeneous suspension solution and followed by continuous stirring and sonication for $30 \mathrm{~min}$ to make the pHA nanoparticle were inserted into the surface of TPU/pHA composite scaffolds. The ultrasonic frequency and ultrasonic efficiency were $20 \mathrm{kHz}$ and $30 \%$. Detailed information for anchoring nanoparticles into the surface of polymer scaffolds using the ultrasonic-assisted approach can be found in our lab's previous study [25]. The pHA-TPU/pHA composite scaffolds with a two-stage modified structure were prepared and washed by deionized water and then freeze-dried for further characterization. A schematic illustration of the fabrication of pHA, TPU/pHA, and pHA-TPU/pHA composite scaffolds is shown in Figure 1.

\subsection{Characterization}

A field-emission scanning electron microscope (FESEM, Nova NanoSEM 450, FEI Company, USA) was used to observe the morphology of pHA, TPU/ pHA, and pHA-TPU/pHA composite scaffolds. A sputter coater (Ace200, Leica, Germany) was used to coat gold onto the samples for examination. A transmission electron microscope (TEM, JME-2100, JEOL Ltd., Japan) was used to observe the morphology of the pHA composite nanoparticles. The surface chemical properties of pHA composite nanoparticles were identified using Fourier transform infrared spectrometry (FTIR, 6700, Nicolet, USA) under the spectral range of 400 to $4000 \mathrm{~cm}^{-1}$. The surface chemical properties of $\mathrm{pHA}$ composite nanoparticles were also analyzed by X-ray diffraction (XRD, D8Advance, Kratos analytical, Chestnut Ridge, USA). Thermo $\neg$ gravi-metric analysis (TGA, STA449F3, Netzsch, Germany) was used to observe the thermal behavior of $\mathrm{pHA}, \mathrm{TPU} / \mathrm{pHA}$, and $\mathrm{pHA}-\mathrm{TPU} / \mathrm{pHA}$ composite scaffolds. The heating rate was $10^{\circ} \mathrm{C} / \mathrm{min}$, with a heating range from 25 to $800^{\circ} \mathrm{C}$ under a nitrogen atmosphere. A contact- angle system (DSA 25, KRUSS, Germany) was used to investigate the hydrophilicity of the pHA, TPU/pHA, and pHA-TPU/ pHA composite scaffolds. Eight samples were used to measure the average value of the samples. 

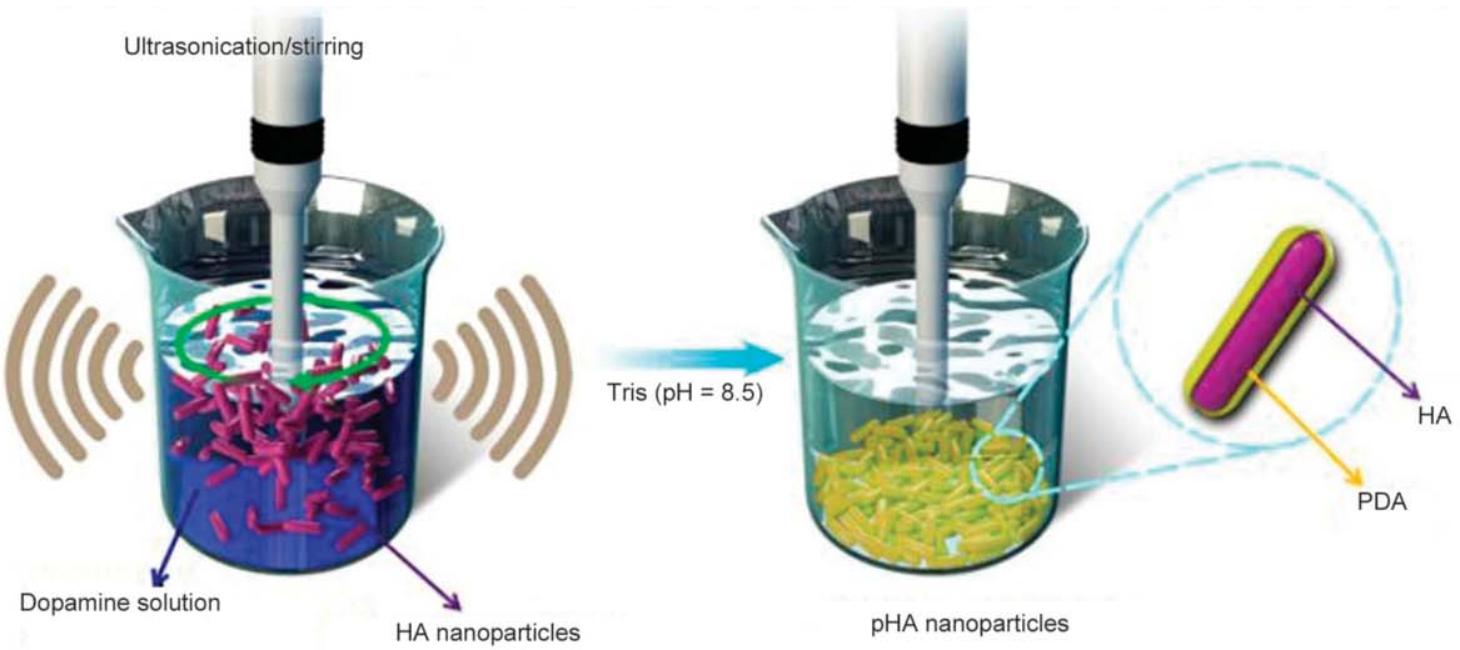

a)
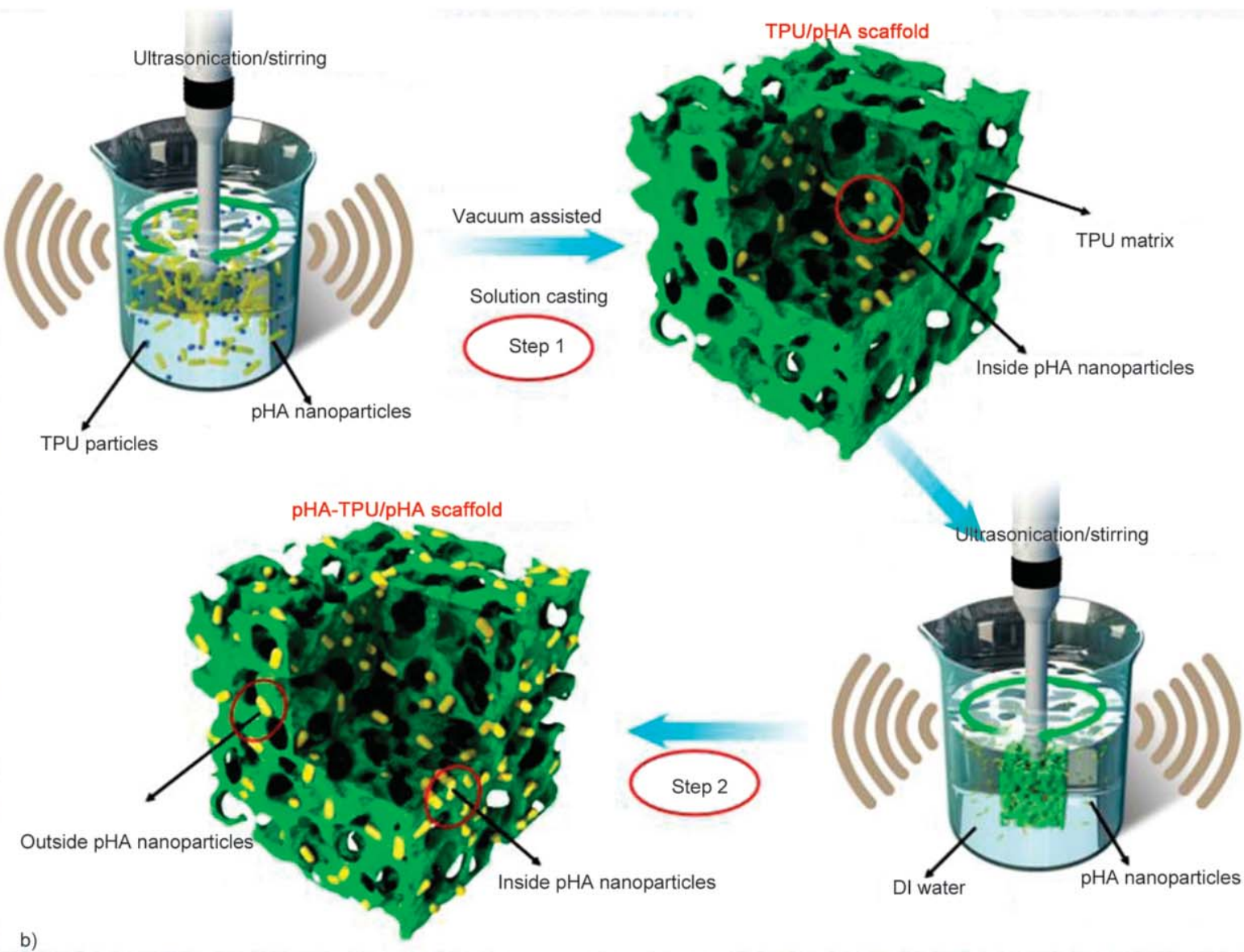

b)

Figure 1. Schematic illustration of (a) pHA, (b) TPU/pHA and pHA-TPU/pHA composite scaffold fabrication.

A solvent replacement method was used to calculate the porosity of the 3-D porous scaffolds. First, the dried 3-D porous scaffolds were measured and referred to as $M_{1}$. Second, the samples were put into an ethanol bath and adsorbed until the absorption equilibrium was reached. The weight of the adsorbed sample was measured and referred to as $M_{2}$. The porosity of the scaffold can be calculated via Equation (1):
Porosity $[\%]=\frac{M_{2}-M_{1}}{\rho V} \cdot 100$

where $V$ and $\rho$ were the volume of the scaffolds and the density of the ethanol, respectively.

The water absorption ability of 3-D porous scaffolds was $37^{\circ} \mathrm{C}$. The original weight of the dry scaffold was measured and referred to as $M_{\mathrm{d}}$. After $24 \mathrm{~h}$ of absorption, the absorbed samples were weighed and 
referred to as $M_{\mathrm{a}}$. The water absorption of the scaffold can be calculated via Equation (2):

Water absorption $[\%]=\frac{M_{\mathrm{a}}-M_{\mathrm{d}}}{M_{\mathrm{a}}} \cdot 100$

A UTM2102 universal testing machine (Suns Technology Stock CO.LTD, China) was used to investigate the static mechanical properties of the 3-D porous scaffolds under a compression test model. Cylindrical samples with a diameter of $8 \mathrm{~mm}$ and a thickness of $10 \mathrm{~mm}$ were prepared. The compression tests were performed under different compression strains of $20,40,60$, and $80 \%$. The crosshead speed was $2 \mathrm{~mm} / \mathrm{min}$. To study the recovery ability of the 3 -D porous scaffolds, a cyclic compression test with a compression strain of $60 \%$ was performed 100 times. Before the above-mentioned compression test, all the samples were first put into phosphate buffered saline (PBS) for $1 \mathrm{~h}$ until the equilibrium wet state was achieved. A DMA 242E instrument (Netzsch, Germany) was applied to investigate the dynamic mechanical properties of the 3 -D porous scaffolds under a compression test model with frequencies of $1,10,50$, and $100 \mathrm{~Hz}$. The range of temperature was from -100 to $100^{\circ} \mathrm{C}$, with a heating speed of $2{ }^{\circ} \mathrm{C} / \mathrm{min}$. Five samples were used to measure the average value of the samples.

\subsection{Cytotoxicity analysis}

An MTT test was used to evaluate cell viability on 3-D porous TPU, TPU/pHA, and $\mathrm{pHA}-\mathrm{TPU} / \mathrm{pHA}$ composite scaffolds. A quantity of $200 \mu$ of a mouse preosteoblast (MC3T3-E1) suspension with a concentration of $10^{4}$ cells $/ \mathrm{ml}$ was injected into the scaffold in a 96-well plate. After culturing for 3, 5, and 7 days under a $5 \% \mathrm{CO}_{2}$ atmosphere at $37^{\circ} \mathrm{C}$, the old culture solution was substituted for a fresh one, and then $20 \mu \mathrm{l}$ of MTT was added into and cultured for another $4 \mathrm{~h}$. Finally, $150 \mu \mathrm{l}$ of dimethyl sulfoxide (DMSO) was added to the plate to replace the culture solution. Before observation, the plate was vibrated for $10 \mathrm{~min}$, then a Micro-plate Reader 450 (Bio-Rad Instruments, USA) was used to analyze the absorbance value at $570 \mathrm{~nm}$. Before SEM observation, the cultured cells on the 3-D porous scaffolds were fixed with $4 \%$ paraformaldehyde at $4{ }^{\circ} \mathrm{C}$ for $6 \mathrm{~h}$. Then a gradient ethanol solution of $50,70,80,90$, 95 , or $100 \%$ was used to dehydrate the cells for 10 min per sample.

\section{Results and discussion}

\subsection{Characterization of pHA nanoparticles}

Figures $2 \mathrm{a}, 2 \mathrm{~b}$ provides TEM images and digital pictures of HA and pHA nanoparticles. It can be seen that the boundary roughness of pHA nanoparticles significantly increased in comparison with that of HA nanoparticles. This is because the crystalline HA nanoparticle was covered by an amorphous PDA coating layer. Also, the color of the pHA nanoparticles changed from white to black after PDA coating. To assess the amount of PDA formed on the surface of the HA nanoparticles, TGA curves of HA, pHA, and PDA nanoparticles are shown in Figure 2c. The lost weight ratios of HA, PDA, and pHA nanoparticles were 2,47 , and $88 \%$, respectively, after the temperature reached $800^{\circ} \mathrm{C}$. Based on the above-mentioned data, the content of PDA in the pHA nanoparticles was calculated to be about $20 \%(\mathrm{PDA} / \mathrm{pHA}, \mathrm{w} / \mathrm{w})$.

Figure 3 a provides the FTIR spectra of $\mathrm{HA}$ and $\mathrm{pHA}$ nanoparticles. For HA nanoparticles, the $\mathrm{PO}_{4}^{3-}\left(v_{4}\right)$ vibrations peaks were observed at 565 and $604 \mathrm{~cm}^{-1}$, along with the $\mathrm{PO}_{4}^{3-}\left(v_{3}\right)$ vibration peak at $1019 \mathrm{~cm}^{-1}$. For pHA nanoparticles, additional peaks at 3445 and $1615 \mathrm{~cm}^{-1}$ were attributed to the $-\mathrm{OH}$ and the benzene ring, respectively, which came from the PDA $[26,27]$. From Figure $3 b$, it can be seen that the peaks at $2 \theta=25.9,31.8,32.8,39.7,46.6,49.4$, and $53.1^{\circ}$, which were indexed to (002), (211), (300), (310), (222), (213), and (004) planes come from the HA nanoparticles were displayed for both HA and HA@PDA nanoparticles [28]. However, it is worth noting that the peaks of (112), (211) and (300) of HA@PDA nanoparticles were slightly transferred into high angles in comparison with that of HA nanoparticles. It may be because the chemical interaction (hydrogen bonding) occurred between HA particles and PDA coating layer. These results demonstrate that the PDA coating layer was successfully introduced onto the surface of the HA nanoparticles.

\subsection{Morphology of TPU, TPU/pHA, and pHA-TPU/pHA composite scaffolds}

Figure 4 provides the SEM images of the TPU, TPU/ pHA, and pHA-TPU/pHA composite scaffolds. For all scaffolds, a 3-D porous structure with highly interconnected pores ranging from $\sim 100$ to $500 \mu \mathrm{m}$ was formed (Figure 4a1-4c1)). Moreover, many smaller pores, with diameters of $10-20 \mu \mathrm{m}$ on the skeleton surface of the scaffolds, were observed 


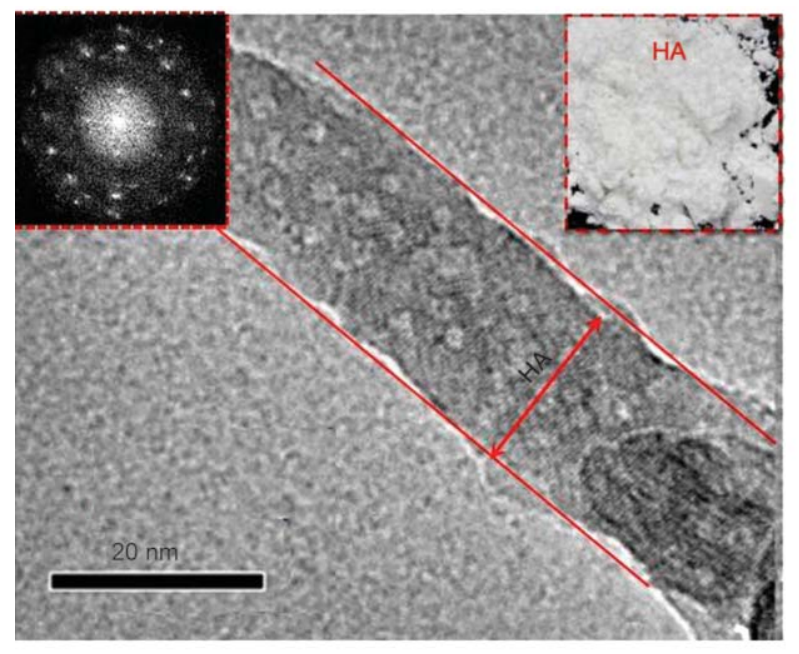

a)

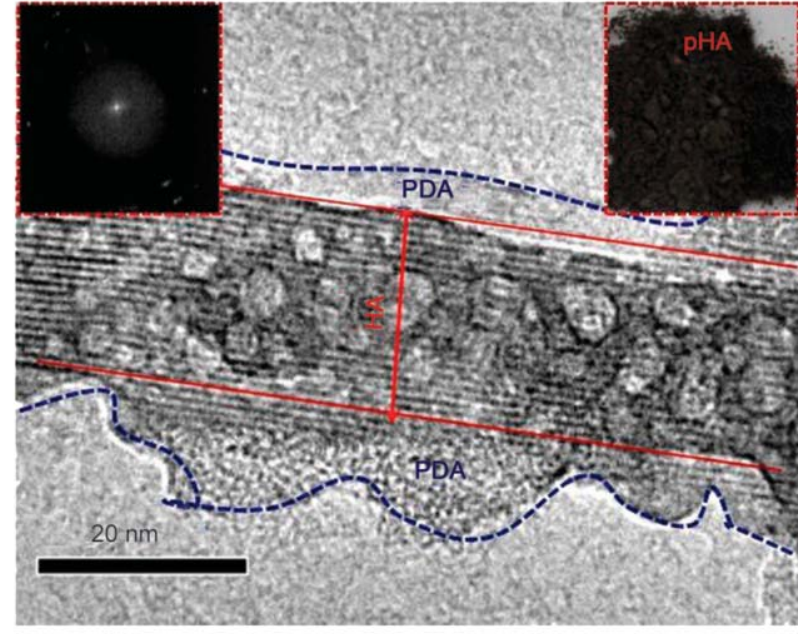

b)

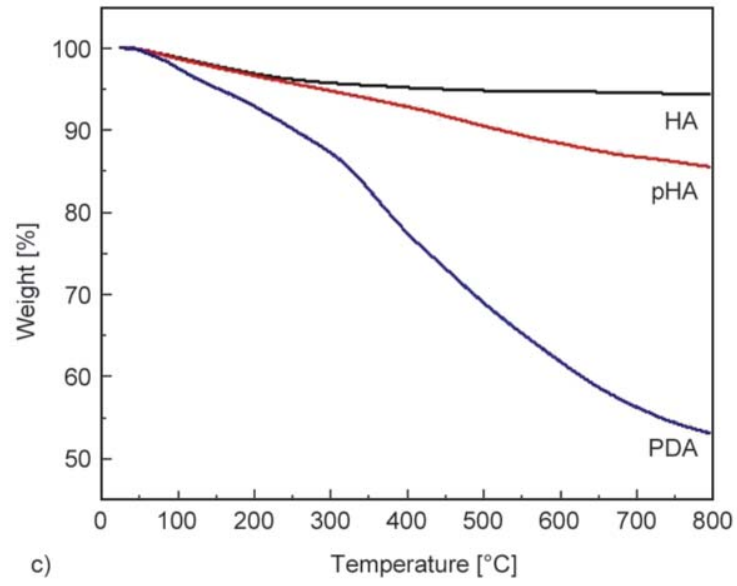

Figure 2. TEM images of (a) HA and (b) pHA nanoparticles. The inset digital pictures in (a) and (b) are the macro-images of HA and pHA nanoparticles. (c) TGA curves of HA, pHA, and PDA nanoparticles.

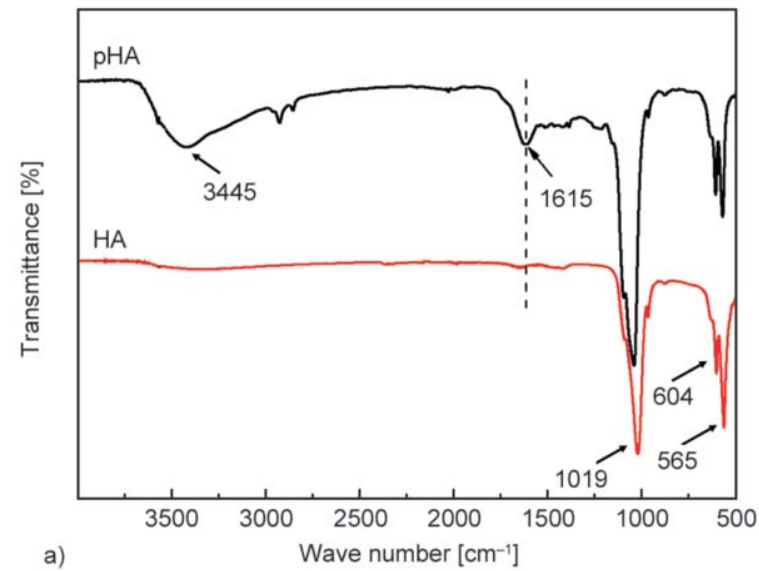

Figure 3. (a) FTIR spectra of HA and pHA nanoparticles. (b) XRD spectra of HA and pHA nanoparticles.

(Figure 4a2-4c2). The excellent 3-D porous network structure and the highly interconnected scaffolds are expected to improve the transplantation of nutrients and cells when used for biological applications.

From the enlarged Figure $4 a 3-4 c 3$, it can be seen that the skeleton surface of the TPU scaffold was smooth, but there were many small particles on the

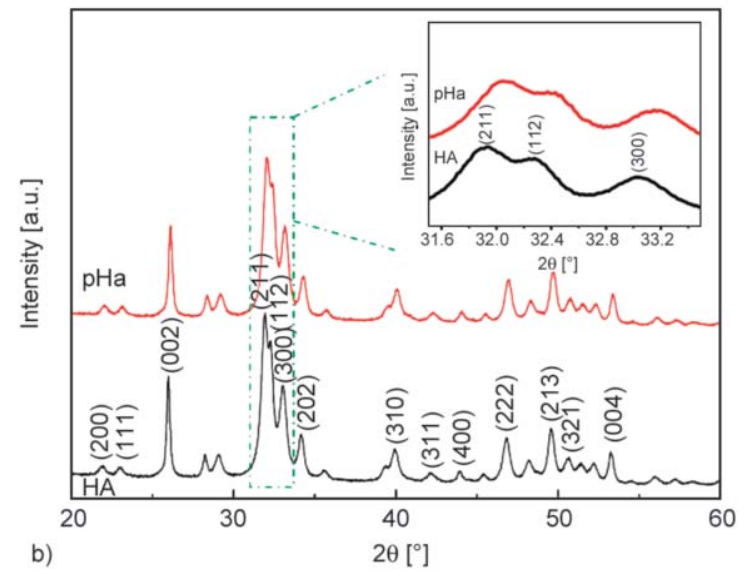

skeleton surface of the TPU/pHA scaffold. This was because some of the pHA nanoparticles were exposed to the exterior surface of the TPU matrix. In contrast, more $\mathrm{pHA}$ nanoparticles on the skeleton surface of the pHA-TPU/pHA scaffold were observed after the ultrasonication-assisted procedure, which made the surface roughness of the pHA-TPU/pHA 


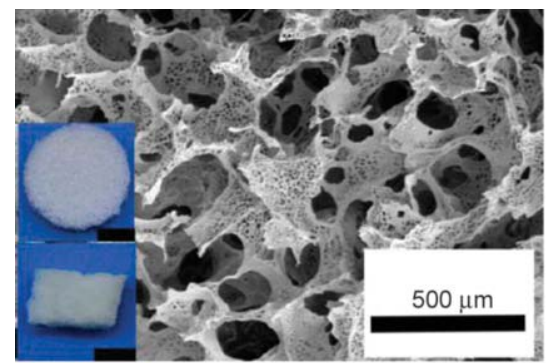

a1)

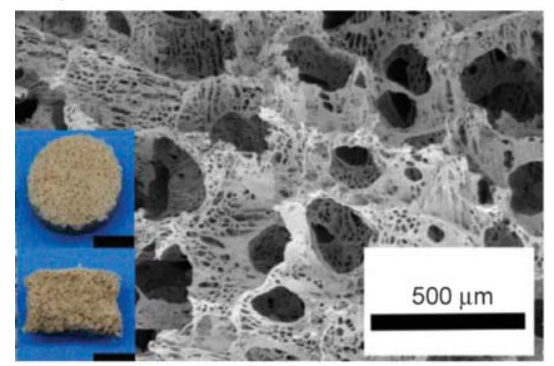

b1)

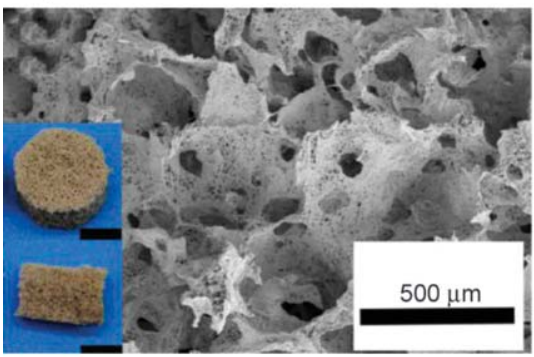

c1)

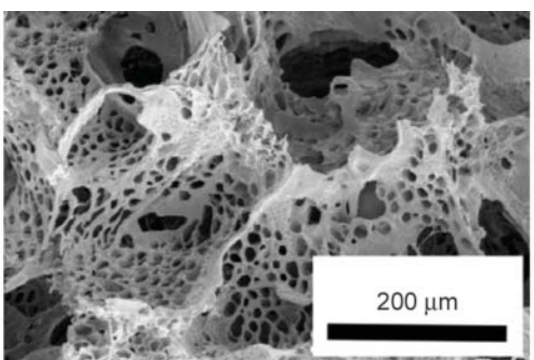

a2)

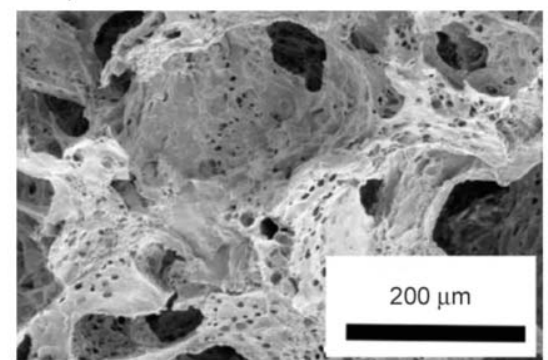

b2)

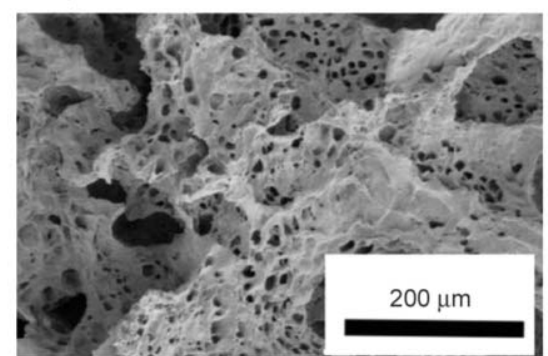

c2)

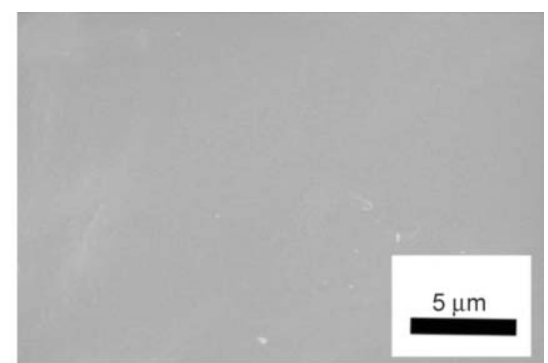

a3)

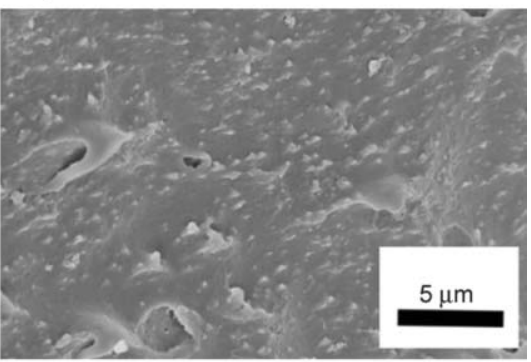

b3)

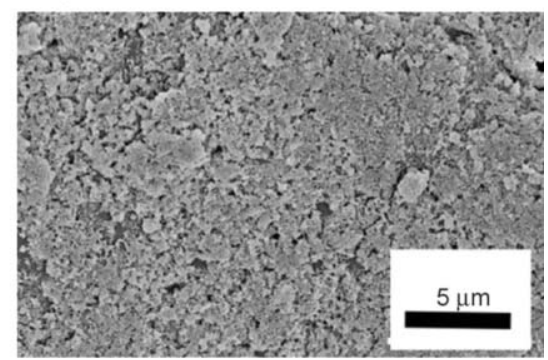

c3)

Figure 4. SEM micrographs of (a1-a3) TPU, (b1-b3) TPU/pHA, and (c1-c3) pHA-TPU/pHA composite scaffolds. The inset digital images in a1, b1, and $\mathrm{c} 1$ are the top view and cross section of TPU, TPU/pHA, and pHA-TPU/pHA composite scaffolds, respectively (The scale bar is $5 \mathrm{~mm}$ ).

scaffold larger than that of the TPU/pHA scaffold. The pHA nanoparticles on the surface of the scaffold are beneficial for improving the interaction between the scaffold and the cells. Moreover, the color of the TPU/pHA and pHA-TPU/pHA scaffolds both changed from white to black due to the addition of pHA nanoparticles, as shown in the inset digital images in Figure 4a1-4c1. These results indicate that the 3-D porous $\mathrm{pHA}-\mathrm{TPU} / \mathrm{pHA}$ scaffolds with two-stage pHA nanoparticle modification were successfully fabricated using the vacuum-assisted solvent casting and ultrasonication-assisted methods. It is noteworthy that the PDA coating layer on the surface of the HA nanoparticles can be effectively used as a transition layer to bond HA nanoparticles to the TPU matrix, and to increase the interfacial bonding force and mechanical properties of the 3 -D porous scaffolds.

The porosity of the scaffold is one of the important factors, because the larger the porosity, the better the reserve and delivery ability. The porosity of the TPU, TPU/pHA, and pHA-TPU/pHA scaffolds were obtained using a previously reported solvent replacement method [29], yielding results of approximately $90.8 \pm 0.4,88.6 \pm 0.3$, and $85.7 \pm 0.6 \%$, respectively. Although the porosity of the pHA-TPU/pHA scaffold slightly decreased in comparison with that of the TPU and TPU/pHA scaffold, it still had a high porosity $(\sim 85 \%)$, which still is beneficial to the transfer of nutrient solution to the cells inside of the 3 -D porous scaffolds.

\subsection{Thermal behavior of TPU, TPU/pHA, and $\mathbf{p H A}-\mathrm{TPU} / \mathrm{pHA}$ composite scaffolds}

The thermal behavior of TPU, TPU/pHA, and pHATPU/pHA composite scaffolds was studied with respect to weight changes and illustrated through the TGA and DTG curves in Figure 5. The loss weight ratio of $\mathrm{pHA}$ was $12 \%$ and tended to remain constant after the temperature reached $800^{\circ} \mathrm{C}$, which was mainly caused by the decomposition of the PDA coating layer. When the loss weight ratio reached $5 \%$, the corresponding temperature was defined as the starting decomposition temperature $\left(T_{5 \%}\right)$. The starting decomposition temperatures of $\mathrm{pHA}-\mathrm{TPU} / \mathrm{pHA}$ 

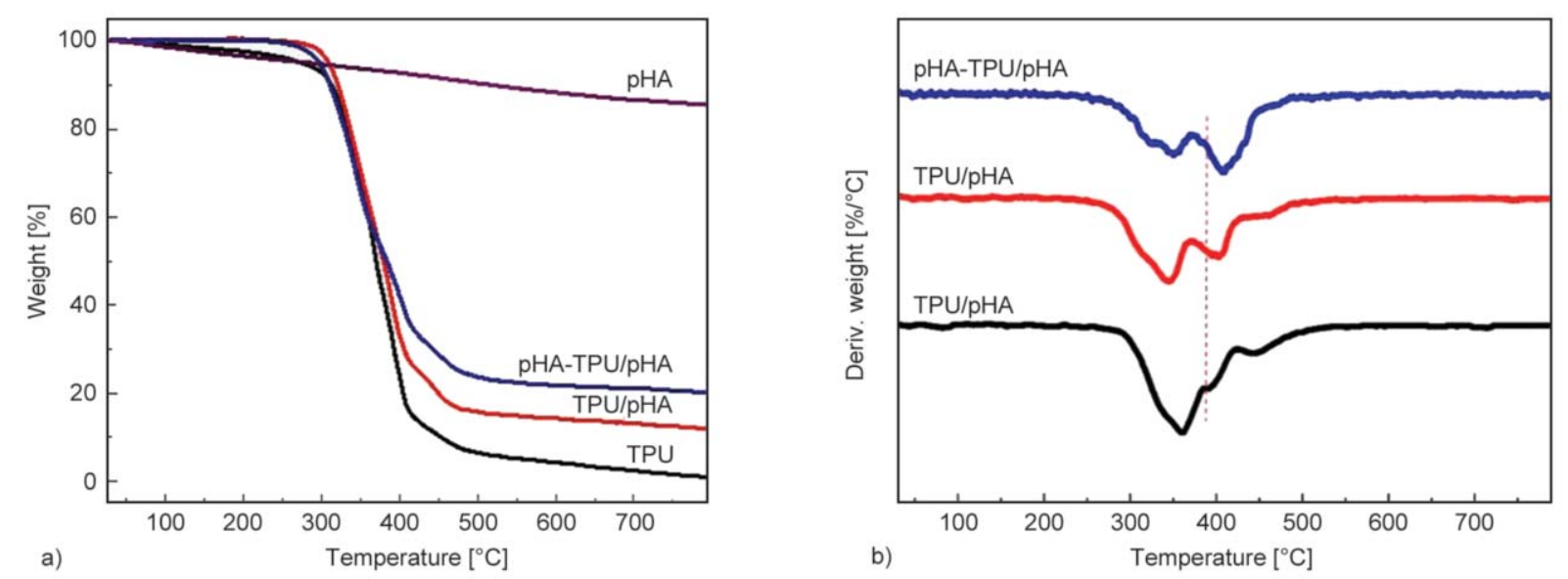

Figure 5. TGA and DTG curves of TPU, TPU/pHA, and pHA-TPU/pHA composite scaffolds.

Table 1. Thermal stability properties of TPU, TPU/pHA, and pHA-TPU/pHA composite scaffolds.

\begin{tabular}{|l|c|c|c|}
\hline \multicolumn{1}{|c|}{ Samples } & $\begin{array}{c}\boldsymbol{T}_{\mathbf{5} \%} \\
{\left[{ }^{\circ} \mathbf{C}\right]}\end{array}$ & $\begin{array}{c}\boldsymbol{T}_{\mathbf{p}} \\
{\left[{ }^{\circ} \mathbf{C}\right]}\end{array}$ & $\begin{array}{c}\text { Residual weight at } \mathbf{8 0 0}^{\circ} \mathbf{C} \\
{[\mathbf{\%}]}\end{array}$ \\
\hline TPU & 259.4 & 388.3 & 2.0 \\
\hline TPU/p-HA & 283.6 & 403.1 & 11.8 \\
\hline p-HA-TPU/p-HA & 295.2 & 408.4 & 20.3 \\
\hline
\end{tabular}

$\left(295.2{ }^{\circ} \mathrm{C}\right)$ and $\mathrm{TPU} / \mathrm{pHA}\left(259.4^{\circ} \mathrm{C}\right)$ scaffolds were higher than that of the TPU $\left(283.6^{\circ} \mathrm{C}\right)$ scaffold (Table 1). This might have been because the pHA nanoparticles had better thermal stability in comparison with the TPU matrix, thus effectively preventing heat transfer into the TPU matrix [30].

All of the scaffolds had a two-stage weight loss. The first stage was in the range of 280 to $380^{\circ} \mathrm{C}$, and the second stage was in the range of 380 to $490^{\circ} \mathrm{C}$. The corresponding temperature of the maximum weight loss rate during the second stage was defined as the maximum decomposition rate temperature $\left(T_{\mathrm{p}}\right)$. The $T_{\mathrm{p}}$ of the $\mathrm{pHA}-\mathrm{TPU} / \mathrm{pHA}$ and TPU/pHA scaffolds was 408.4 and $403.1^{\circ} \mathrm{C}$, respectively, which increased in

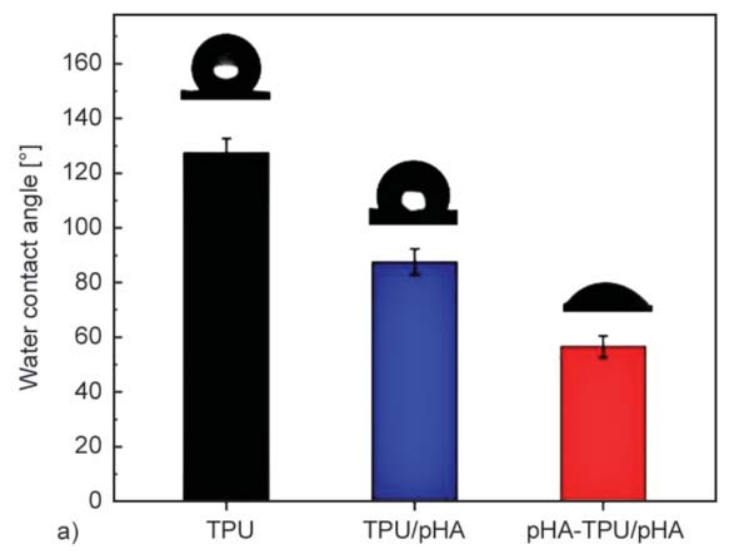

comparison with that of the TPU scaffold $\left(388.3{ }^{\circ} \mathrm{C}\right)$. These results suggest that the thermal stability of a 3-D porous $\mathrm{pHA}-\mathrm{TPU} / \mathrm{pHA}$ scaffold can be significantly increased by adding pHA nanoparticles.

\subsection{Hydrophilicity and water adsorption capacity}

The hydrophilicity and water absorption capacity of scaffolds are one of the main factors affecting cell behaviors, such as morphology, attachment, and proliferation. The water contact angle is widely used to assess the surface hydrophilicity of materials. As shown in Figure 6a, the water contact angle of the TPU scaffold decreased significantly, from 127.5 \pm 5.1 to $87.6 \pm 4.9^{\circ}$, for the TPU/pHA scaffold after introducing pHA nanoparticles in the interior of the TPU matrix. It further decreased to $59.2 \pm 3.9^{\circ}$ for $\mathrm{pHA}$ TPU/pHA composite scaffolds due to the further addition of pHA nanoparticles on the exterior of the TPU matrix. This is mainly because pHA nanoparticles have excellent hydrophilic properties due to their rich hydrophilic hydrogen and amino groups

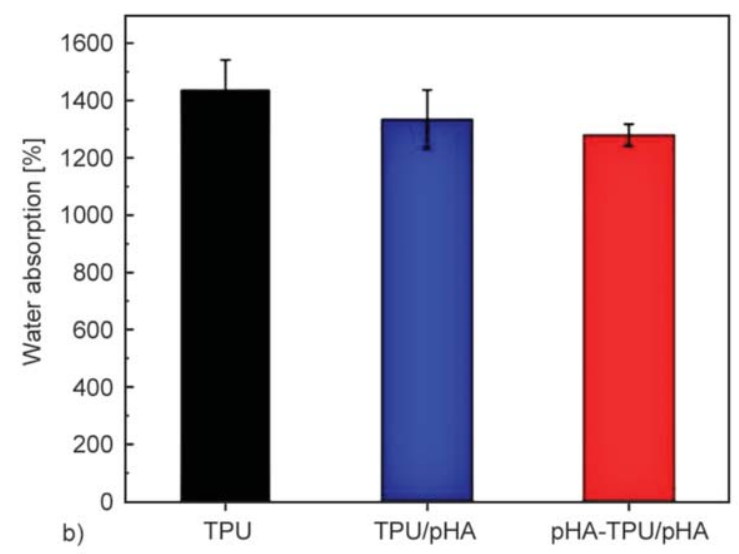

Figure 6. The hydrophilicity (a) and water absorption (b) of TPU, TPU/pHA, and pHA-TPU/pHA composite scaffolds. 
that come from the PDA coating layer. And another reason is that the surface roughness increased when lots of pHA nanoparticles were inserted onto the surface of the scaffolds, which results in a decrease in water contact angle. This indicated that the hydrophilicity of 3-D porous $\mathrm{pHA}$-TPU/pHA scaffolds can be significantly increased by adding pHA nanoparticles to the interior and exterior of the scaffold.

The water absorption of TPU, TPU/pHA, and pHA-TPU/pHA composite scaffolds were $1440 \pm 101$, $1334 \pm 102$, and $1279 \% \pm 37 \%$, respectively, as shown in Figure $6 \mathrm{~b}$. The water absorption of the scaffolds decreased slightly with an increase in the addition of pHA, but they still retained their high water absorption. This suggests that scaffold porosity is the main factor affecting water absorption because high porosity can create more water storage space. These results indicate that the excellent hydrophilicity and water adsorption capacity of pHA-TPU/pHA composite scaffolds will be a benefit to promote their bioactive properties and further to improve the interaction between cells and scaffolds.

\subsection{Mechanical properties}

In order to observe the macro recoverability of pHA-TPU/pHA composite scaffold, the compression-release test was performed by using $100 \mathrm{~g}$ weight. From Figure $7 \mathrm{a}$, it can be seen that the thickness of pHA-TPU/pHA composite scaffold decreased by $60 \%$ when $100 \mathrm{~g}$ weight was placed atop of it. And its thickness quickly regained the original height when the load was removed. This demonstrates that the scaffold possesses good recoverability. The typical stressstrain curves of TPU, TPU/pHA, and pHA-TPU/pHA scaffolds are shown in Figure $7 \mathrm{~b}$, and their corresponding compressive modulus and compressive strengths at a strain of 50\% are shown in Figure 7c. It can be seen that the compressive modulus and compressive strength of the TPU scaffold significantly improved from $18.6 \mathrm{kPa}$ and 10.2 to $26.5 \mathrm{kPa}$ and $19.3 \mathrm{kPa}$ for TPU $/ \mathrm{pHA}$, and further increased to 30.3 and $24.5 \mathrm{kPa}$ for the $\mathrm{pHA}-\mathrm{TPU} / \mathrm{pHA}$ scaffold. This was mainly because the pHA nanoparticles possessed excellent mechanical properties; therefore, the mechanical properties of the TPU/pHA scaffold increased after adding pHA nanoparticles to the TPU matrix. Furthermore, the mechanical properties of the pHA-TPU/pHA scaffold further increased when more pHA nanoparticles were added onto the surface of the scaffold due to the formation of a rigid continual phase on the surface of the scaffold. Moreover, the TPU/pHA and pHA-TPU/pHA composite scaffolds showed good interfacial adhesion between the filler (pHA nanoparticles) and the TPU matrix because the PDA coating layer on the surface of the HA nanoparticles can be acted like a glue-like transition layer, which led to increased mechanical properties.

To evaluate the recoverability of the TPU, TPU/pHA, and pHA-TPU/pHA scaffolds, the compression stressstrain curves under dynamic environments with different strains of 20, 40, 60, and 80\% are shown in Figure $7 \mathrm{~d}-7 \mathrm{f}$. It can be seen that, for all scaffolds, the deformation shapes quickly recovered to their original shapes after the load was released. This further confirmed that all of the scaffolds possessed good recoverability due to the prominent elasticity of the TPU matrix and the high porosity of the scaffolds. When the scaffolds endured a high compressive strain above $60 \%$, the compressive stress of the scaffolds sharply increased. This was because, when the stress was below $60 \%$, the deformation of the scaffolds was mainly characterized by the pore structure being compressed. When the compressive strain was above $60 \%$, however, the pores completely collapsed and the skeleton of the scaffolds came into contact with itself, which made the compressive stress increase drastically.

The compression stress-strain curves under dynamic environments with different numbers of cycles at a strain of $60 \%$ for TPU, TPU/pHA, and pHA-TPU/ pHA scaffolds are provided in Figure 7g-7i. The hysteresis loss in the stress-strain curves can be used to explain the energy dissipation when the scaffolds endure loading/unloading cycles. For all scaffolds, the hysteresis loss decreased as the cycle number increased. It nearly stabilized after 50 cycles, which indicated that all of the scaffolds possessed good fatigue resistance and low energy loss in repetitive loading/unloading cycles. These results demonstrated that the pHA-TPU/pHA scaffold not only had better strength and stiffness in comparison with the TPU and TPU/pHA scaffolds due to the addition of pHA nanoparticles in the inside and outside of the TPU matrix, but it also retained its excellent elasticity and recoverability.

The dynamic mechanical properties of the TPU, TPU/pHA, and pHA-TPU/pHA composite scaffolds are provided in Figure 8; the test frequency was $1 \mathrm{~Hz}$. For composite materials, the filler properties, filler dispersion within the matrix, matrix properties, and the interfacial adhesion between the filler and the 


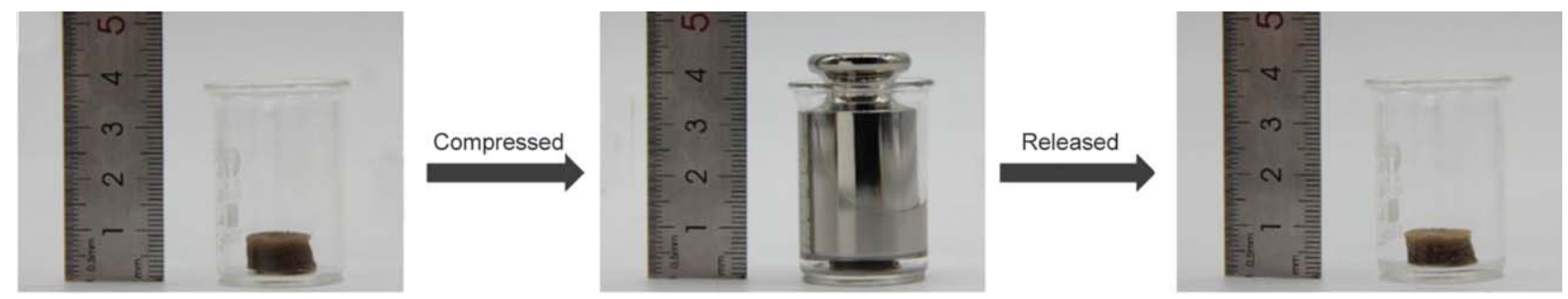

a)
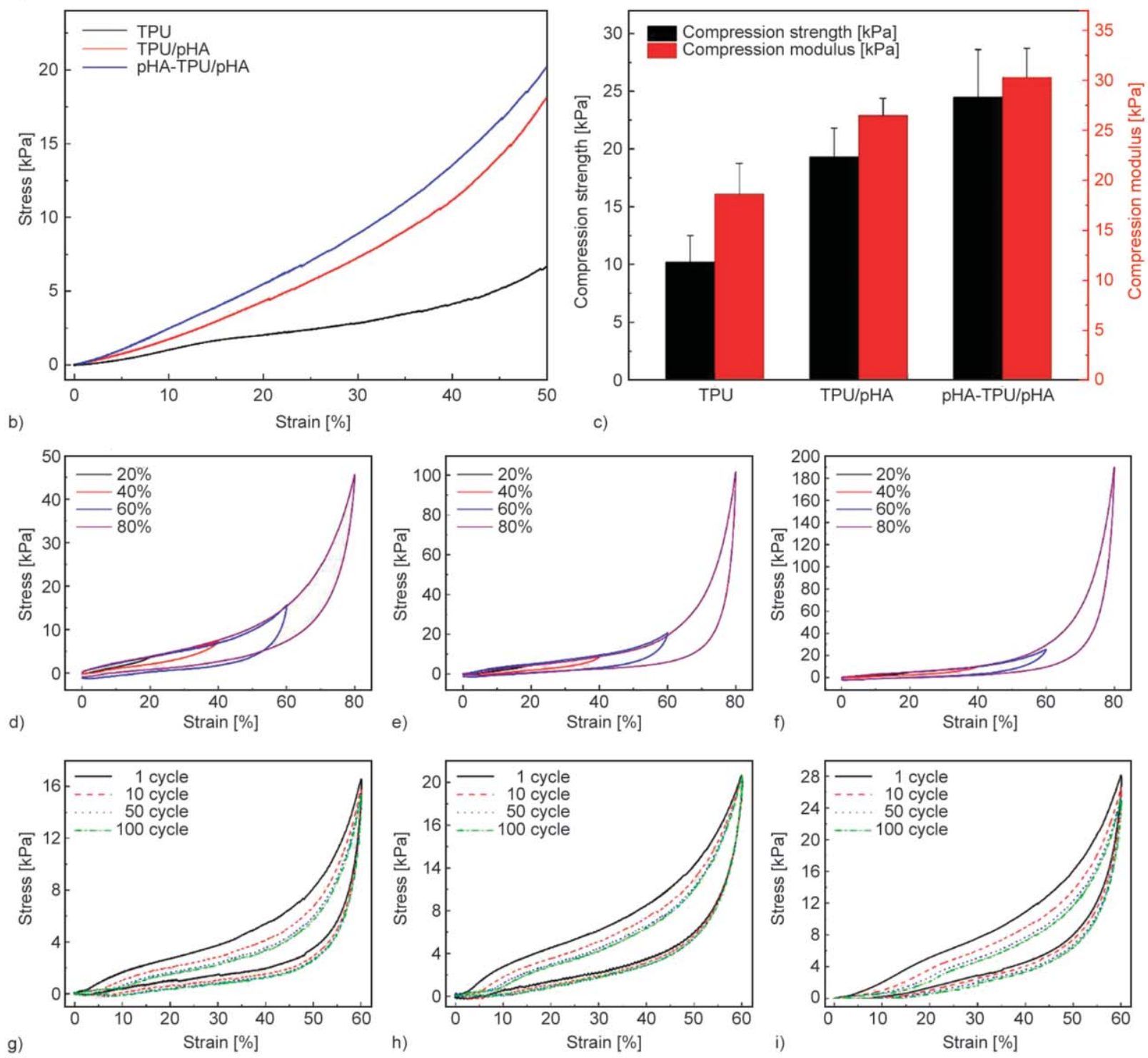

Figure 7. (a) Photographs of a compressed-released test for pHA-TPU/pHA scaffold. (b) Typical stress-strain curves of TPU, TPU/pHA, and pHA-TPU/pHA scaffolds. (c) Compressive modulus and compressive strength at a strain of 50\%. (d-f) Compression stress-strain curves at strains from 20 to $80 \%$ for (d) TPU, (e) TPU/pHA, and (f) pHA-TPU/ pHA scaffolds. (g-i) Compression stress-strain curves with different numbers of cycles at a strain of $60 \%$ for (g) TPU, (h) TPU/pHA, and (i) pHA-TPU/pHA scaffolds.

matrix were the major factors affecting their dynamic mechanical properties [31]. From Figure 8a, it can be seen that the storage modulus of all scaffolds decreased as the temperature increased, and decreased rapidly at a temperature range of -50 to $10^{\circ} \mathrm{C}$. This was attributed to the fact that the mobility of the molecular chains increased with increasing temperature, thus resulting in lower storage modulus. The storage modulus of the TPU/pHA and pHA-TPU/pHA scaffolds were larger than that of the TPU scaffold. The pHA-TPU/pHA scaffold had the largest storage modulus. There are two reasons to explain this phenomenon: (1) the pHA is a rigid nanoparticle and possesses a high modulus, which can restrict the 

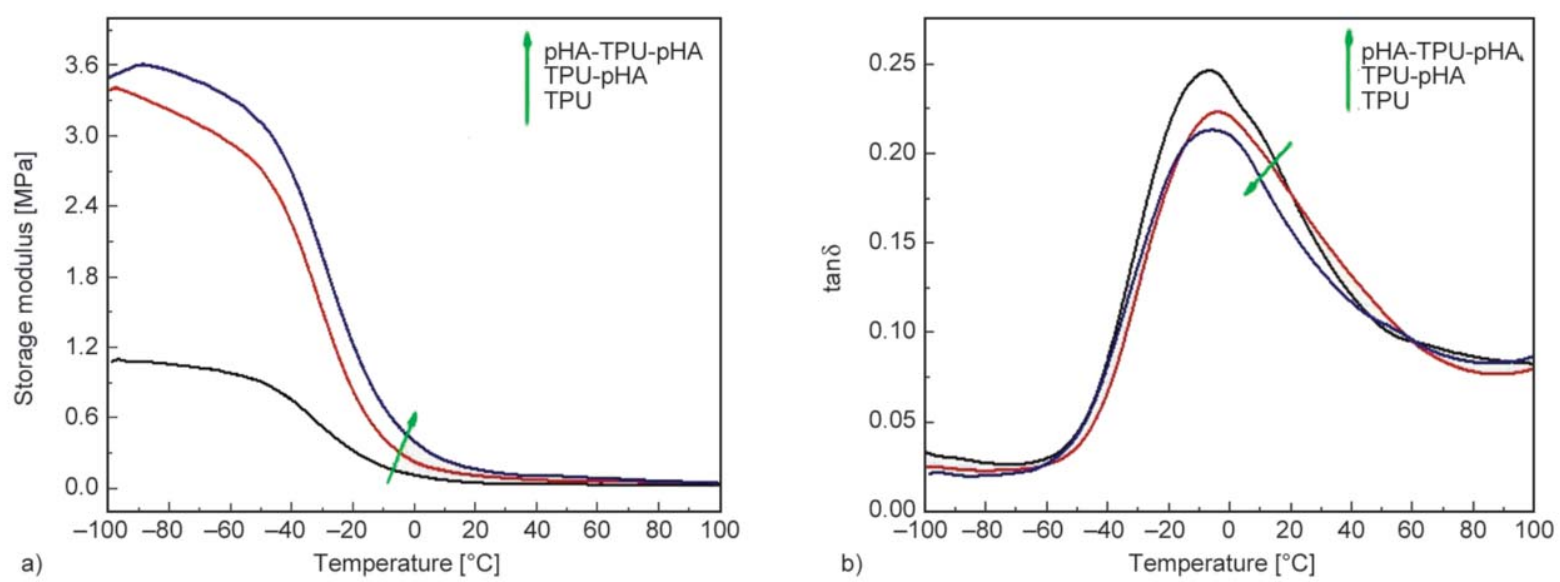

Figure 8. Dynamic mechanical properties of TPU, TPU/pHA, and pHA-TPU/pHA composite scaffolds. (a) Storage modulus versus temperature curves. (b) $\tan \delta$ versus temperature curves.

movement and flexibility of the molecular chains of the TPU matrix; and/or (2) the PDA coating as a transitional layer increases the interaction between $\mathrm{pHA}$ nanoparticles and the TPU matrix, leading to an increase in the interfacial binding force and limiting the movement of the molecular chains.

The damping coefficient $(\tan \delta)$ versus temperature curves of TPU, TPU/pHA, and pHA-TPU/pHA composite scaffolds were provided in Figure $8 \mathrm{~b}$. The corresponding temperature of the maximum value of $\tan \delta$ was usually defined as the glass transition temperature $\left(T_{\mathrm{g}}\right)$ of the material. From Figure $8 \mathrm{~b}$, it can be seen that the $T_{\mathrm{g}}$ of TPU/pHA and $\mathrm{pHA}-\mathrm{TPU} / \mathrm{pHA}$ scaffolds was slightly higher than that of the TPU scaffold. The higher $T_{\mathrm{g}}$ indicated that free motion among the molecular chains occurred at a higher temperature, resulting in higher mechanical properties for materials [32]. This result is consistent with the result obtained from Figure $7 \mathrm{c}$. Besides, for composite materials, $\tan \delta$ can be applied to evaluate the energy dissipation caused by irreversible deformation due to the movement of molecular chains. A smaller $\tan \delta$ represents better interfacial adhesion while a larger $\tan \delta$ represents poorer interfacial adhesion. It is worth noting that the maximum value of $\tan \delta$ for both the TPU/pHA and pHA-TPU/pHA composite scaffolds were small in comparison with that of the TPU scaffold, which indicates that there were strong interfacial binding forces between the pHA nanoparticles and the TPU matrix.

\subsection{Morphology of MC3T3-E1 cells on composite scaffolds}

Figure 9 displays the morphology of MC3T3-E1 cells cultured on TPU, TPU/pHA, and pHA-TPU/pHA composite scaffolds for 3 and 7 days. After seeding for 3 days, the MC3T3-E1 cells were able to adhere to all scaffolds, showing a normal round and oval shape. In particular, the cells cultured on the pHA-TPU/pHA composite scaffolds show filopodia and lamellipodia. After culturing for 5 days, the cells cultured on all scaffolds showed partial stretching, especially the cells cultured on the pHA-TPU/pHA composite scaffolds, which completely stretched along the surface of the scaffold and presented more filopodia and lamellipodia compared to the TPU/pHA and TPU scaffolds. This result indicated that the cell biocompatibility of pHA-TPU/pHA composite scaffolds with two-stage modification significantly improved due to the introduction of pHA nanoparticles to both the inside and outside of the scaffold.

\subsection{Viabilities of MC3T3-E1 cells on composite scaffolds}

An MTT assay was applied to quantitatively evaluate the viabilities of MC3T3-E1 cells on the TPU, TPU/pHA, and pHA-TPU/pHA composite scaffolds with culture times of 3,5 , and 7 days, as shown in Figure 10. It can be seen that the cells cultured on the TPU/pHA and pHA-TPU/pHA composite scaffolds were remarkably larger than those cultured on the TPU scaffold, which suggests that the introduction of pHA nanoparticles effectively increased the cell biocompatibility. Even more remarkably, the cell number cultured on the $\mathrm{pHA}-\mathrm{TPU} / \mathrm{pHA}$ composite scaffolds showed a drastic improvement in comparison with those cultured on the TPU/pHA composite scaffold. This was because more pHA nanoparticles were introduced onto the surface of the pHA-TPU/ pHA composite scaffold, resulting in a significant 


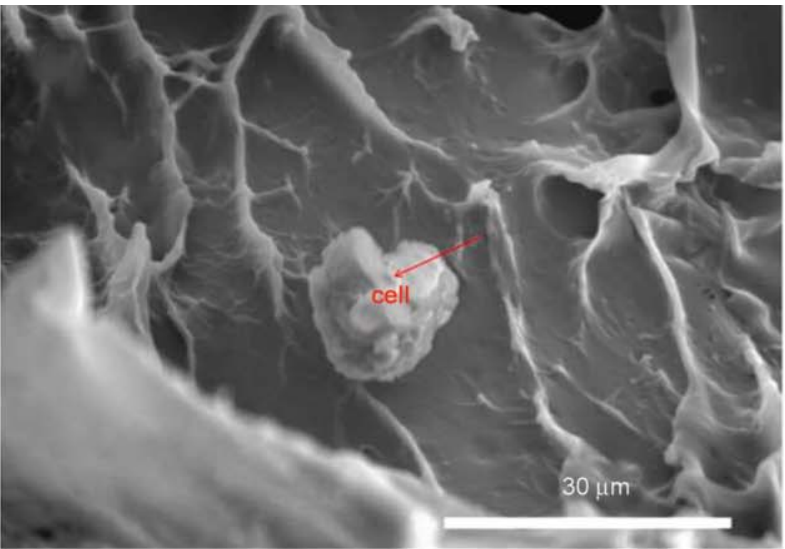

a)

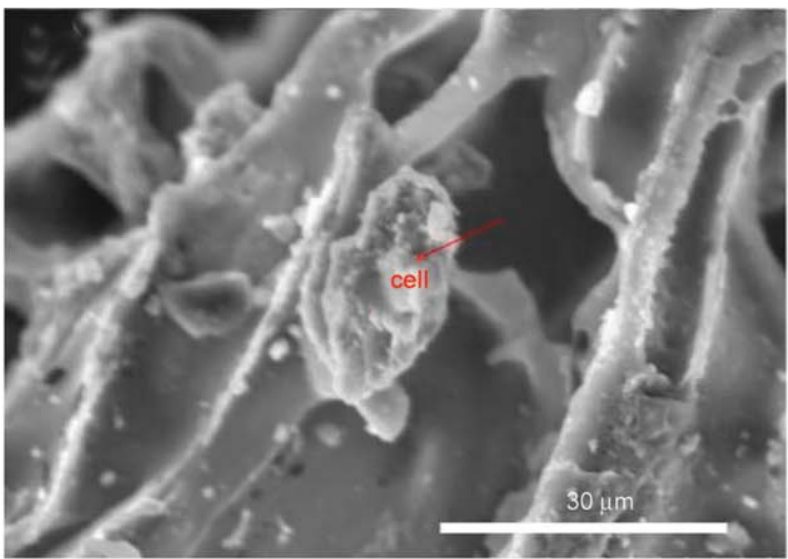

c)

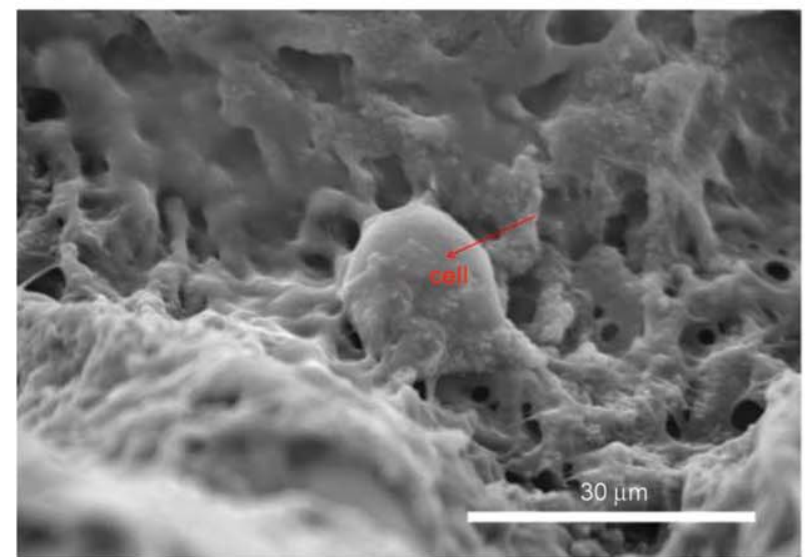

e)

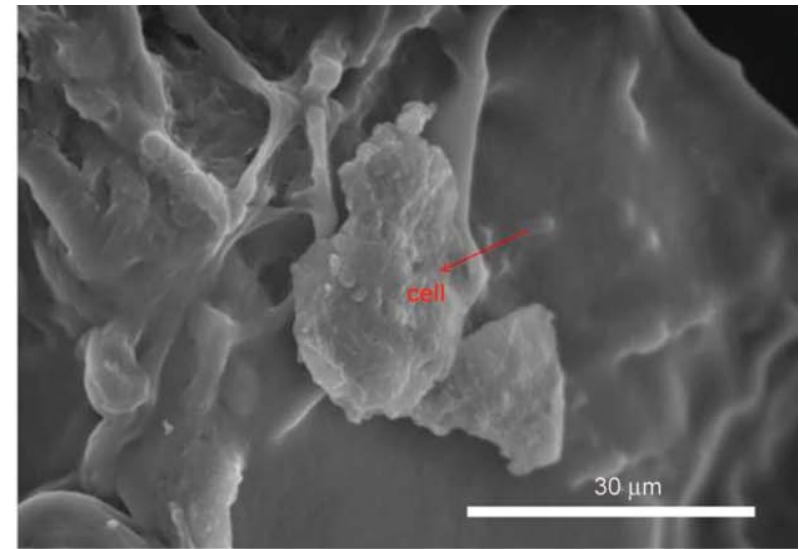

b)

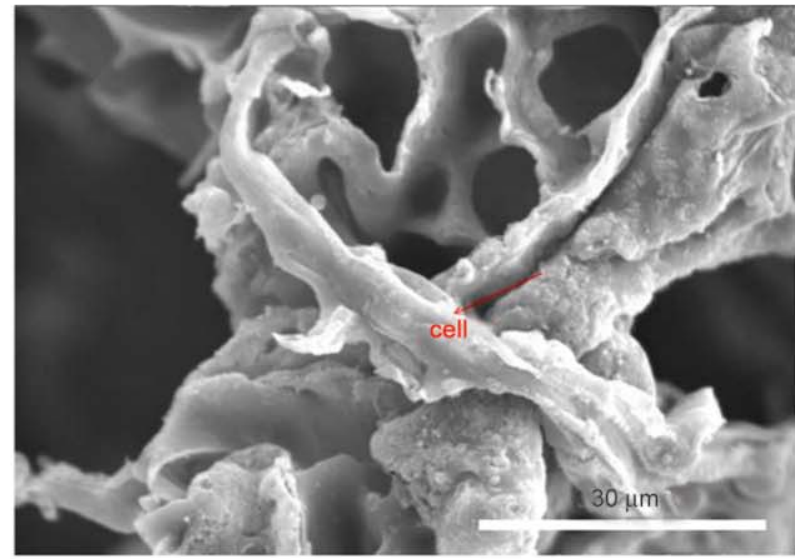

d)

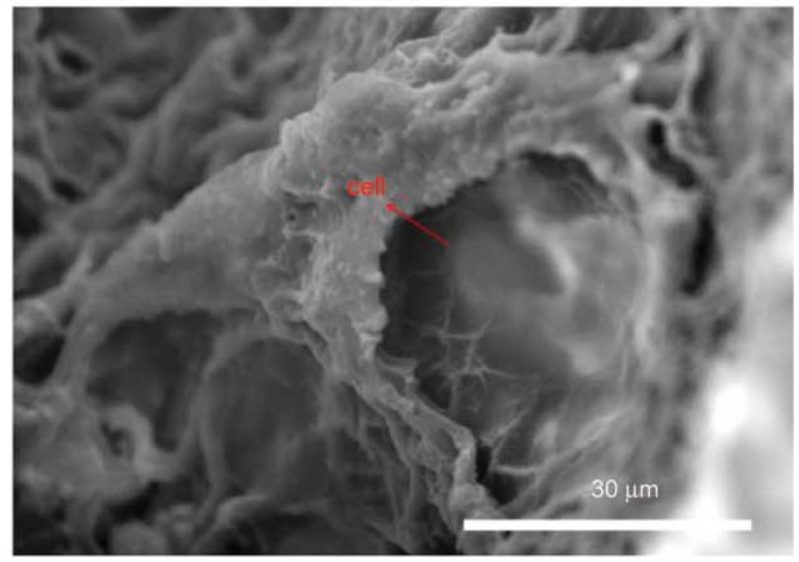

f)

Figure 9. Morphology of MC3T3-E1 cells cultured for (a, c, e) 3 days and (b, d, f) 5 days on (a, b) TPU, (c, d) TPU/pHA, and (e, f) pHA-TPU/pHA composite scaffolds.

increase in interactions between the cells and the scaffold. This result also confirmed that the biocompatibility of the pHA-TPU/pHA composite scaffolds with two-stage modification significantly improved due to the introduction of pHA nanoparticles to both the inside and outside of the scaffold.

\section{Conclusions}

PDA coating layers were first introduced onto the surface of HA nanoparticles by a facile method to improve their bioactivity. TEM, FTIR, and TGA measurements confirmed that the pHA composite nanoparticles were successfully prepared and were further used to modify 3-D porous TPU scaffolds. The functional $\mathrm{pHA}-\mathrm{TPU} / \mathrm{pHA}$ composite scaffolds with two-stage modification in both the interior and external of the scaffold were successfully fabricated. The pHA-TPU/pHA composite scaffolds possessed an excellent interconnected network structure, excellent hydrophilicity, high porosity of $85.7 \%$, high water 


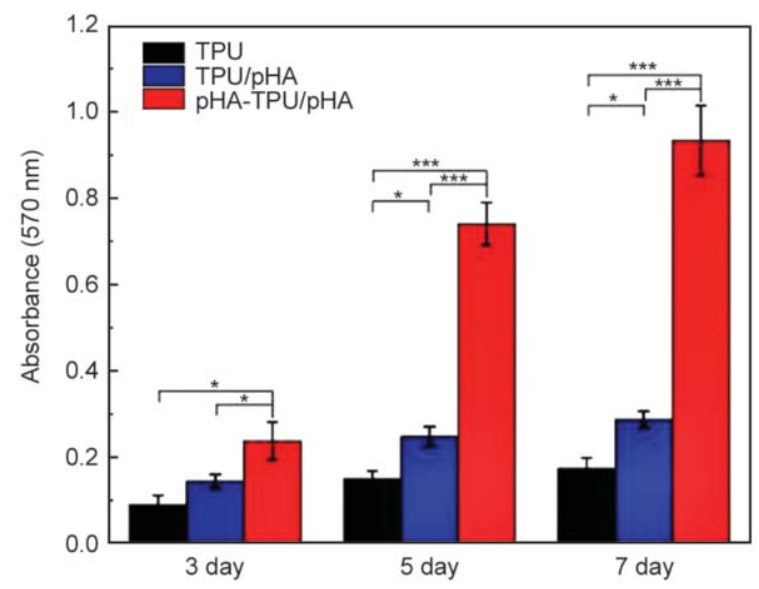

Figure 10. The MTT values of MC3T3-E1 on TPU, TPU/ pHA, and pHA-TPU/pHA composite scaffolds with culture times of 3,5 , and 7 days. $\left({ }^{*} p<0.05\right.$ and $* * * p<0.001)$.

absorption of $1279 \%$, and large pore size ranging from 100 to $500 \mu \mathrm{m}$. The $\mathrm{pHA}$-TPU/pHA composite scaffolds not only exhibited higher strength and stiffness compared with TPU scaffolds, they also retained their excellent elasticity and recoverability, and demonstrated remarkable and comprehensive mechanical properties. The significant enhancement of the adhesion and viability of MC3T3-E1 cells cultured on pHA-TPU/pHA composite scaffolds demonstrated that they had good biocompatibility and accelerated cell adhesion and proliferation. These results demonstrate that the functional $\mathrm{pHA}-\mathrm{TPU} / \mathrm{pHA}$ composite scaffolds possess great potential for use as tissue engineering scaffolds.

\section{Acknowledgements}

The authors would like to acknowledge the financial support of the Outstanding Young Scientific Research Personnel Training Plan in the Colleges and Universities of Fujian Province (Grant No. GY-Z160146), the Research Fund of Fujian University of Technology (Grant No. GY-Z15091, GY-Z160121), the Program of New Century Excellent Talents in the University of Fujian Province (Grant No. GYZ17065), the External Cooperative Projects of Fujian Province (Grant No. 2018I0001), the Young Teachers Education Research Project (Grant No. JAT170377), the National Natural Science Foundation of China (Grant No. 51303027, 61605027), the Wisconsin Institute for Discovery (WID) at the University of Wisconsin-Madison, China Scholarship Council, and Opening Funding of School of Pharmacy of Fujian Medical University.

\section{References}

[1] Langer R., Vacanti J. P.: Tissue engineering. Science, 260, 920-926 (1993).

https://doi.org/10.1126/science.8493529
[2] Pollok J. M., Vacanti J. P.: Tissue engineering. Seminars in Pediatric Surgery, 5, 191-196 (1996).

[3] Glimcher M. J.: The nature of the mineral phase in bone: Biological and clinical implications. in 'Metabolic bone disease and clinically related disorders' (eds.: Avioli L. V., Krane S. M.) Academic, San Diego, 2350 (1998).

https://doi.org/10.1016/B978-012068700-8/50003-7

[4] Crane G. M., Ishaug S. L., Mikos A. G.: Bone tissue engineering. Nature Medicine, 1, 1322-1324 (1996). https://doi.org/10.1038/nm1295-1322

[5] Dang M., Saunders L., Niu X., Fan Y., Ma P.: Biomimetic delivery of signals for bone tissue engineering. Bone Research, 6, 205-216 (2018).

https://doi.org/10.1038/s41413-018-0025-8

[6] Wang L., He S., Wu X., Liang S., Mu Z., Wei J., Deng F., Deng Y., Wei S.: Polyetheretherketone/nano-fluorohydroxyapatite composite with antimicrobial activity and osseointegration properties. Biomaterials, 35, 6758-6775 (2014).

https://doi.org/10.1016/j.biomaterials.2014.04.085

[7] Zhao M., Li H., Liu X., Wei J., Ji J., Yang S., Hu Z., Wei S.: Response of human osteoblast to $n$-HA/PEEKquantitative proteomic study of bio-effects of nano-hydroxyapatite composite. Scientific Reports, 6, 2283222845 (2016).

https://doi.org/10.1038/srep22832

[8] Mi H-Y., Jing X., Yilmaz G., Hagerty B. S., Enrquez E., Turng L-S.: In situ synthesis of polyurethane scaffolds with tunable properties by controlled crosslinking of tri-block copolymer and polycaprolactone triol for tissue regeneration. Chemical Engineering Journal, 348, 786-798 (2018).

https://doi.org/10.1016/j.cej.2018.04.198

[9] Pina S., Oliveira J. M., Reis R. L.: Natural-based nanocomposites for bone tissue engineering and regenerative medicine: A review. Advanced Materials, 27, 11431169 (2015).

https://doi.org/10.1002/adma.201403354

[10] Huang G., Li F., Zhao X., Ma Y., Li Y., Lin M., Jin G., $\mathrm{Lu}$ T., Genin G. M., Xu F.: Functional and biomimetic materials for engineering of the three-dimensional cell microenvironment. Chemical Reviews, 117, 1276412850 (2017).

https://doi.org/10.1021/acs.chemrev.7b00094

[11] Izquierdo-Barba I., Santos-Ruiz L., Becerra J., Feito M. J., Fernandez-Villa D., Serrano M. C., Diaz-Güemes I., Fernandez-Tomé B., Enciso S., Sánchez-Margallo F. M., Monopoli D., Afonso H., Portolés M. T., Arcos D., Vallent-Regí M.: Synergistic effect of Si-hydroxyapatite coating and VEGF adsorption on $\mathrm{Ti}_{6} \mathrm{Al}_{4} \mathrm{~V}$-ELI scaffolds for bone regeneration in an osteoporotic bone environment. Acta Biomaterialia, 83, 456-466 (2019). https://doi.org/10.1016/j.actbio.2018.11.017

[12] Shao N., Guo J., Guan Y., Zhang H., Li X., Chen X., Zhou D., Huang Y.: Development of organic/inorganic compatible and sustainably bioactive composites for effective bone regeneration. Biomacromolecules, 19, 3637-3648 (2018).

https://doi.org/10.1021/acs.biomac.8b00707 
[13] Du J., Zuo Y., Lin L., Huang D., Niu L., Wei Y., Wang K., Lin Q., Zou Q., Li Y.: Effect of hydroxyapatite fillers on the mechanical properties and osteogenesis capacity of bio-based polyurethane composite scaffolds. Journal of the Mechanical Behavior of Biomedical Materials, 88, 150-159 (2018).

https://doi.org/10.1016/j.jmbbm.2018.08.028

[14] Yang W., Both S., Zuo Y., Birgani T. B., Habibovic P., Li Y., Jansen J. A., Yang F.: Biological evaluation of porous aliphatic polyurethane/hydroxyapatite composite scaffolds for bone tissue engineering. Journal of Biomedical Materials Research, 103, 2251-2259 (2015). https://doi.org/10.1002/jbm.a.35365

[15] Li L., Zuo Y., Zou Q., Yang B., Lin L., Li J., Li Y.: Hierarchical structure and mechanical improvement of an n-HA/GCO-PU composite scaffold for bone regeneration. ACS Applied Materials and Interfaces, 7, 22618 22629 (2015).

https://doi.org/10.1021/acsami.5b07327

[16] Boissard C. I. R., Bourban P-E., Tami A. E., Alini M., Eglin D.: Nanohydroxyapatite/poly(ester urethane) scaffold for bone tissue engineering. Acta Biomaterialia, 5, 3316-3327 (2009).

https://doi.org/10.1016/j.actbio.2009.05.001

[17] Vasile E., Popescu L. M., Piticescu R. M., Burlacu A., Buruiana T.: Physico-chemical and biocompatible properties of hydroxyapatite based composites prepared by an innovative synthesis route. Materials Letters, 79, 8588 (2012).

https://doi.org/10.1016/j.matlet.2012.03.099

[18] Bunpetch V., Zhang X., Li T., Lin J., Maskwikiti E. P., Wu Y., Cai D., Li J., Zhang S., Wu C., Ouyang H. W.: Silicate-based bioceramic scaffolds for dual-lineage regeneration of osteochondral defect. Biomaterials, 192, 323-333 (2019). https://doi.org/10.1016/j.biomaterials.2018.11.025

[19] Cu Z., Zheng Z., Su C., Si J., Wang Q., Chen W.: Porous 3-D thermoplastic polyurethane (TPU) scaffold modified with hydroxyapatite (HA) nanoparticles using an ultrasonic method. Journal of Materials Science, 54, 11231-11242 (2019). https://doi.org/10.1007/s10853-019-03683-6

[20] Lee H., Dellatore S. M., Miller W. M., Messersmith P. B.: Mussel-inspired surface chemistry for multifunctional coatings. Science, 318, 426-430 (2007). https://doi.org/10.1126/science.1147241

[21] Liu Y., Ai K., Lu L.: Polydopamine and its derivative materials: Synthesis and promising applications in energy, environmental, and biomedical fields. Chemical Reviews, 114, 5057-5115 (2014). https://doi.org/10.1021/cr400407a

[22] Ryu J. H., Messersmith P. B., Lee H.: Polydopamine surface chemistry: A decade of discovery. ACS Applied Materials and Interfaces, 10, 7523-7540 (2018).

https://doi.org/10.1021/acsami.7b19865
[23] Zheng Z., Cui Z., Si J., Yu S., Wang Q., Chen W., Turng L-S.: Modification of 3-D porous hydroxyapatite/thermoplastic polyurethane composite scaffolds for reinforcing interfacial adhesion by polydopamine surface coating. ACS. Omega, 4, 6382-6391 (2019). https://doi.org/10.1021/acsomega.9b00404

[24] Zeng S., Cui Z., Yang Z., Si J., Wang Q., Wang X., Peng K., Chen W.: Characterization of highly interconnected porous poly(lactic acid) and chitosan-coated poly(lactic acid) scaffold fabricated by vacuum-assisted resin transfer molding and particle leaching. Journal of Materials Science, 51, 9958-9970 (2016). https://doi.org/10.1007/s10853-016-0203-2

[25] Ye J., Si J., Cui Z., Wang Q., Peng K., Chen W., Peng X., Chen S-C.: Surface modification of electrospun TPU nanofiber scaffold with CNF particles by ultrasoundassisted technique for tissue engineering. Macromolecular Materials and Engineering, 302, 1700277/11700277/9 (2017).

https://doi.org/10.1002/mame.201700277

[26] Zhang Y-G., Zhu Y-J., Chen F., Lu B-Q.: Dopaminemodified highly porous hydroxyapatite microtube networks with efficient near-infrared photothermal effect, enhanced protein adsorption and mineralization performance. Colloids and surfaces B: Biointerfaces, 159, 337-348 (2017).

https://doi.org/10.1016/j.colsurfb.2017.07.093

[27] Zhang K., Wang Y., Sun T., Wang B., Zhang H.: Bioinspired surface functionalization for improving osteogenesis of electrospun polycaprolactone nanofibers. Langmuir, 34, 15544-15550 (2018).

https://doi.org/10.1021/acs.langmuir.8b03357

[28] Li Q., Li M., Zhu P., Wei S.: In vitro synthesis of bioactive hydroxyapatite using sodium hyaluronate as a template. Journal of Materials Chemistry, 22, 20257-20265 (2012). https://doi.org/10.1039/C2JM33624C

[29] Yin L., Fei L., Cui F., Tang C., Yin C.: Superporous hydrogels containing poly(acrylic acid-co-acrylamide)/Ocarboxymethyl chitosan interpenetrating polymer networks. Biomaterials, 28, 1258-1266 (2006). https://doi.org/10.1016/j.biomaterials.2006.11.008

[30] Lima P. A., Resende C. X., de Almeida Soares G. D., Anselme K., Almeida L. E.: Preparation, characterization and biological test of 3D-scaffolds based on chitosan, fibroin and hydroxyapatite for bone tissue engineering. Materials Science and Engineering: C, 33, 3389-3395 (2013).

https://doi.org/10.1016/j.msec.2013.04.026

[31] Yu J., Huang X., Wu C., Wu X., Wang G., Jiang P.: Interfacial modification of boron nitride nanoplatelets for epoxy composites with improved thermal properties. Polymer, 53, 471-480 (2012).

https://doi.org/10.1016/j.polymer.2011.12.040

[32] Liu Y., Fang Y., Qian J., Liu Z., Yang B., Wang X.: Bioinspired polydopamine functionalization of carbon fiber for improving the interfacial adhesion of polypropylene composites. RSC Advance, 5, 107652-107661 (2015). https://doi.org/10.1039/C5RA20045H 